\title{
A Widespread, Clumpy Starburst in the Isolated Ongoing Dwarf Galaxy Merger dm1647+21
}

\author{
G. C. Privon ${ }^{1}$ (i), S. Stierwalt ${ }^{2}$, D. R. Patton ${ }^{3}$ (1D), G. Besla ${ }^{4}$, S. Pearson ${ }^{5}$ (1), \\ M. Putman ${ }^{5}$, K. E. Johnson ${ }^{2}$ (i), N. Kallivayalii ${ }^{2}$, and S. Liss ${ }^{2}$
}

(TiNy Titans)

\footnotetext{
${ }^{1}$ Instituto de Astrofśica, Facultad de Física, Pontificia Universidad Católica de Chile, Casilla 306, Santiago 22, Chile; gprivon@astro.puc.cl

${ }_{2}^{2}$ Department of Astronomy, University of Virginia, 530 McCormick Road, Charlottesville, VA 22904, USA

${ }^{3}$ Department of Physics and Astronomy, Trent University, 1600 West Bank Drive, Peterborough, ON K9L 0G2, Canada

${ }^{4}$ Department of Astronomy, University of Arizona, 933 North Cherry Avenue, Tucson, AZ 85721, USA

${ }^{5}$ Department of Astronomy, Columbia University, 550 West 120th Street, New York, NY 10027, USA

Received 2017 March 30; revised 2017 July 27; accepted 2017 August 7; published 2017 September 1
}

\begin{abstract}
Interactions between pairs of isolated dwarf galaxies provide a critical window into low-mass hierarchical, gasdominated galaxy assembly and the build-up of stellar mass in low-metallicity systems. We present the first Very Large Telescope/Multi Unit Spectroscopic Explorer (VLT/MUSE) optical integral field unit (IFU) observations of the interacting dwarf pair dm1647+21 selected from the TiNy Titans survey. The $\mathrm{H} \alpha$ emission is widespread and corresponds to a total unobscured star formation rate (SFR) of $0.44 M_{\odot} \mathrm{yr}^{-1}$, which is 2.7 times higher than the SFR inferred from Sloan Digital Sky Survey (SDSS) data. The implied specific SFR (sSFR) for the system is elevated by more than an order of magnitude above non-interacting dwarfs in the same mass range. This increase is dominated by the lower-mass galaxy, which has a sSFR enhancement of $>50$. Examining the spatially resolved maps of classic optical line diagnostics, we find that the interstellar medium (ISM) excitation can be fully explained by star formation. The velocity field of the ionized gas is not consistent with simple rotation. Dynamical simulations indicate that the irregular velocity field and the stellar structure is consistent with the identification of this system as an ongoing interaction between two dwarf galaxies. The widespread, clumpy enhancements in the star formation in this system point to important differences in the effect of mergers on dwarf galaxies, compared to massive galaxies; rather than the funneling of gas to the nucleus and giving rise to a nuclear starburst, starbursts in low-mass galaxy mergers may be triggered by large-scale ISM compression, and thus may be more distributed.
\end{abstract}

Key words: galaxies: dwarf - galaxies: individual (SDSS J164710.66+210514.5, SDSS J164711.12+210514.8) galaxies: interactions - galaxies: ISM - galaxies: starburst

\section{Dwarf-Dwarf Interactions and dm1647+21}

Dynamical interactions and mergers between massive galaxies are known to effect dramatic structural changes in galaxies, in addition to triggering starbursts and active galactic nuclei (e.g., Toomre \& Toomre 1972; Barnes 1988; Sanders et al. 1988; Stierwalt et al. 2013). These events are at least partially responsible for the morphological and color transformation of massive galaxies from late- to early-type galaxies (e.g., Hopkins et al. 2008). These interactions are well studied, particularly at low-redshift.

However, comparatively little is known about the physical processes driven by interactions between low-mass galaxies. Observations of low-redshift dwarf galaxy interactions and mergers probe the hierarchical assembly in systems with lowmetallicity gas and whose baryonic component is dominated by atomic gas. The Large Magellanic Cloud/Small Magellianic Cloud (LMC/SMC) pair provides a close-up view of an interacting pair of dwarfs, but the environment of the Milky Way makes it difficult to isolate the effects of the interaction (e.g., Besla et al. 2012).

In order to study galaxy assembly at low-mass, lowmetallicity, and high gas fraction, we have identified a sample of Sloan Digital Sky Survey (SDSS)-selected paired and unpaired dwarf galaxies, including both isolated systems and systems near a massive host. The TiNy Titans (TNT; Stierwalt et al. 2015) survey enables us to disentangle environmental effects (the proximity to a massive host) and dynamical effects (the interaction with a similar mass companion) in the evolution of low-mass galaxies. The paired sources are thought to be physically associated, based on their projected separations $(\Delta R<50 \mathrm{kpc})$ and relative velocities $\left(\Delta v<300 \mathrm{~km} \mathrm{~s}^{-1}\right)$. The paired and unpaired sources are further separated into those isolated from a massive host (massive galaxies must be at $\Delta R>1.5 \mathrm{Mpc}, \Delta v>1000 \mathrm{~km} \mathrm{~s}^{-1}$ ) and those associated with a massive host.

Fully understanding the influence mergers and interactions have on dwarf galaxies requires detailed studies of individual systems to spatially resolve the activity and dynamics. We present the first results from Very Large Telescope (VLT) Multi Unit Spectroscopic Explorer (MUSE; Bacon et al. 2010) observations of dwarf pairs. Where appropriate, we adopt the WMAP9 cosmology $\left(H_{0}=69.3 \mathrm{~km} \mathrm{~s}^{-1} \mathrm{Mpc}^{-1}, \Omega_{M}=0.286\right.$, $\Omega_{\text {vacuum }}=0.714$; Hinshaw et al. 2013).

\subsection{Overview of $d m 1647+21$}

The object of the present study, dm1647+21, is a dwarf pair at $16^{\mathrm{h}} 47^{\mathrm{m}} 10^{\mathrm{s}} .9+21^{\mathrm{d}} 05^{\mathrm{m}} 14.5$, with a redshift of $z=0.0090994$ $\left(D_{L}=39.6 \mathrm{Mpc}\right.$ and $\left.0.189 \mathrm{kpc} /{ }^{\prime \prime}\right)$. The pair consists of the SDSS spectroscopic objects SDSS J164710.66+210514.5 and SDSS J164711.12+210514.8, which have a projected linear 
separation of $5 \mathrm{kpc}$ and a velocity difference of $27 \mathrm{~km} \mathrm{~s}^{-1}$ (Stierwalt et al. 2015). The system also meets the above isolation criteria from a massive galaxy. Using the multi-band SDSS photometry, we compute a total stellar mass for the system of $9.0 \times 10^{7} M_{\odot}$ (calculated as described in Stierwalt et al. 2015) with a mass ratio between the two components of 4.6:1. Based on the SDSS spectroscopic measurements and an extrapolation to the entire galaxy the system has an estimated star formation rate of $0.15 M_{\odot} \mathrm{yr}^{-1}$ (Brinchmann et al. 2004). The metallicity inferred for the system is $Z=7.84$ (Tremonti et al. 2004).

The pair has a nearby companion galaxy at a similar redshift, UGC 10549, which has a stellar mass of $2.4 \times 10^{8} M_{\odot}$ (from SDSS DR12, using the Maraston et al. 2009 method). UGC 10549 does not formally meet the association criterion for TNT pairs, with a projected separation to dm1647+21 just over $50 \mathrm{kpc}$, and thus was not initially identified as being associated. However, considering the proximity of this object to $\mathrm{dm} 1647+21$, this may be a potential dwarf-only group similar to those found by Stierwalt et al. (2017).

Using integral field unit (IFU) observations of dm1647+21 (Section 2), we explore the activity and kinematics of the system (Section 3) to test the dwarf-dwarf interaction scenario for the system and to compare the activity to those of massive galaxy mergers (Section 4).

\section{MUSE IFU Observations of $\operatorname{dm} 1647+21$}

A total of $2895 \mathrm{~s}$ of on-source integration were obtained for dm1647+21 with MUSE in clear conditions on 2016 September 5 in three exposures with the Wide Field Mode and nominal wavelength coverage (480-930 nm). The data were reduced and calibrated with the ESO Reflex pipeline software (Freudling et al. 2013). A sky background was subtracted using emission-free regions of the cube.

The 1 arcminute extent of the MUSE field of view (FOV) corresponds to a linear scale of $11.3 \mathrm{kpc}$ at the distance of $\mathrm{dm} 1647+21$, and covers the full optical extent of the system. The cube has a resolution of $\sim 0.90(170 \mathrm{pc})$ and $\sim 0.75$ $(140 \mathrm{pc})$ at $500 \mathrm{~nm}$ and $800 \mathrm{~nm}$, respectively. The spectral resolution varies from $R=1750-3750$, from the blue to the red.

\subsection{Creation of Broadband and Line Images}

Using the MUSE data cube and the SDSS $r$ filter transmission curve, we construct a broadband image for $\mathrm{dm} 1647+21$ (Figure 1, top left). We also detect emission from the important optical diagnostic lines: $\mathrm{H} \beta$, the [O III] doublet, $\mathrm{H} \alpha$, the [N II] doublet, and the [S II] doublet (Table 1, Figure 1). The [O I] line at $6300 \AA$ was redshifted to the location of a sky line and we cannot identify a clear detection. Line images were created using adjacent line-free channels to subtract the continuum underneath the line, then summing the channels visually identified to contain line emission. The resulting images were masked by convolving each image with a Gaussian with $\sigma=1.5$ pixels and determining the rms value of an emission-free portion of the image. Pixels in the unsmoothed line image with values less than three times this rms value were masked.

\section{Optical Properties of $\mathrm{dm} 1647+21$}

\subsection{Optical Continuum Emission}

The deep $r$-band image generated from the MUSE data shows low-significance extended emission which may be an extension of tidal material from the lower-mass galaxy. The background level of the image is dominated by striations which likely result from imperfect illumination correction for the IFU modules. These limit our per-pixel $3 \sigma$ background sensitivity to $\mu_{r} \sim 25.5 \mathrm{mag} \operatorname{arcsec}^{-2}\left(\sim 2 \mathrm{mag} \operatorname{arcsec}^{-2}\right.$ fainter than the SDSS $r$ image for this field). At this level, we see no evidence for an underlying envelope of extended continuum emission that would suggest this is a single, irregular galaxy. Future deep optical continuum observations (e.g., Duc et al. 2015) would be useful to confirm the possible faint tidal emission and to search for evidence of an extended stellar envelope. The blue dashed line in Figure 1 shows the approximate dividing line adopted for the photometric separation of the two component galaxies.

\subsection{Star Formation}

From the system-integrated $\mathrm{H} \alpha$ flux, we compute a $\mathrm{H} \alpha$ luminosity of $3.5 \times 10^{40} \mathrm{erg} \mathrm{s}^{-1}$.

Assuming an intrinsic $\mathrm{H} \alpha / \mathrm{H} \beta$ ratio of 2.86 and utilizing the LMC average extinction curve from Gordon et al. (2003), we compute $A_{\mathrm{H} \alpha} \sim 0.2-1.4$, with a system-averaged value of $0.51{ }^{6}$ Using this calculated extinction value, the intrinsic $\mathrm{H} \alpha$ luminosity is $5.6 \times 10^{40} \mathrm{erg} \mathrm{s}^{-1}$. Using the Kennicutt (1998) relation for the star formation rate, we compute an SFR of $0.44 M_{\odot} \mathrm{yr}^{-1}$ (corrected for extinction) for the system, which is a factor of 2.7 higher than the total SFR estimated from the extrapolation of the SDSS spectroscopy to the whole system (e.g., Brinchmann et al. 2004). The observed equivalent width of the $\mathrm{H} \alpha$ emission is $118 \AA$, so this system belongs to the population of starbursting dwarfs ( $\mathrm{H} \alpha$ equivalent width (EQW) > $100 \AA$ A Lee et al. 2009b).

The dm1647+21 pair was detected with the Wide-field Infrared Survey Explorer (WISE), with a W4 $(22 \mu \mathrm{m})$ flux of $6.43 \mathrm{mJy}$ (Wright et al. 2010). If this flux is entirely due to star formation, this corresponds to an obscured SFR of $0.04 M_{\odot}$ $\mathrm{yr}^{-1}$ (using the calibration from Chang et al. 2015), which is an order of magnitude smaller than the SFR inferred from $\mathrm{H} \alpha$. This indicates that the majority of the star formation is occurring in regions not heavily obscured by dust and is consistent with the relatively low extinction inferred from the Balmer decrement. Thus, we are confident that the $\mathrm{H} \alpha$ image is indicative of the true distribution of star formation in $\mathrm{dm} 1647+21$. Summing the obscured and unobscured SFR estimates, we find a total SFR of $0.48 M_{\odot} \mathrm{yr}^{-1}$. Considering the measured stellar mass from SDSS photometry and the total SFR, the specific SFR (sSFR) is $5.3 \times 10^{-9} \mathrm{yr}^{-1}$, corresponding to a mass-doubling time of 190 Myr. The northern galaxy has $\sim 40 \%$ of the $\mathrm{H} \alpha$ flux and the southern system has the remainder. Considering the 4.6:1 stellar mass ratio, the sSFR for the lower-mass galaxy is a factor of 7 larger than the more massive galaxy.

The brightest $\mathrm{H} \alpha$ clump in the system has an observed $\mathrm{H} \alpha$ flux of $23.3 \times 10^{-15} \mathrm{erg} \mathrm{s}^{-1} \mathrm{~cm}^{-2}$ (measured within a 1.15 circular aperture), accounting for $12 \%$ of the total $\mathrm{H} \alpha$ flux for

\footnotetext{
6 The extinction MWd the subsequent extinction-corrected SFRs are relatively robust to the choice of a LMC versus MW extinction curve. Using a Milky Way extinction curve, as in Lee et al. (2009a), produces values within $10 \%$ of those presented here.
} 


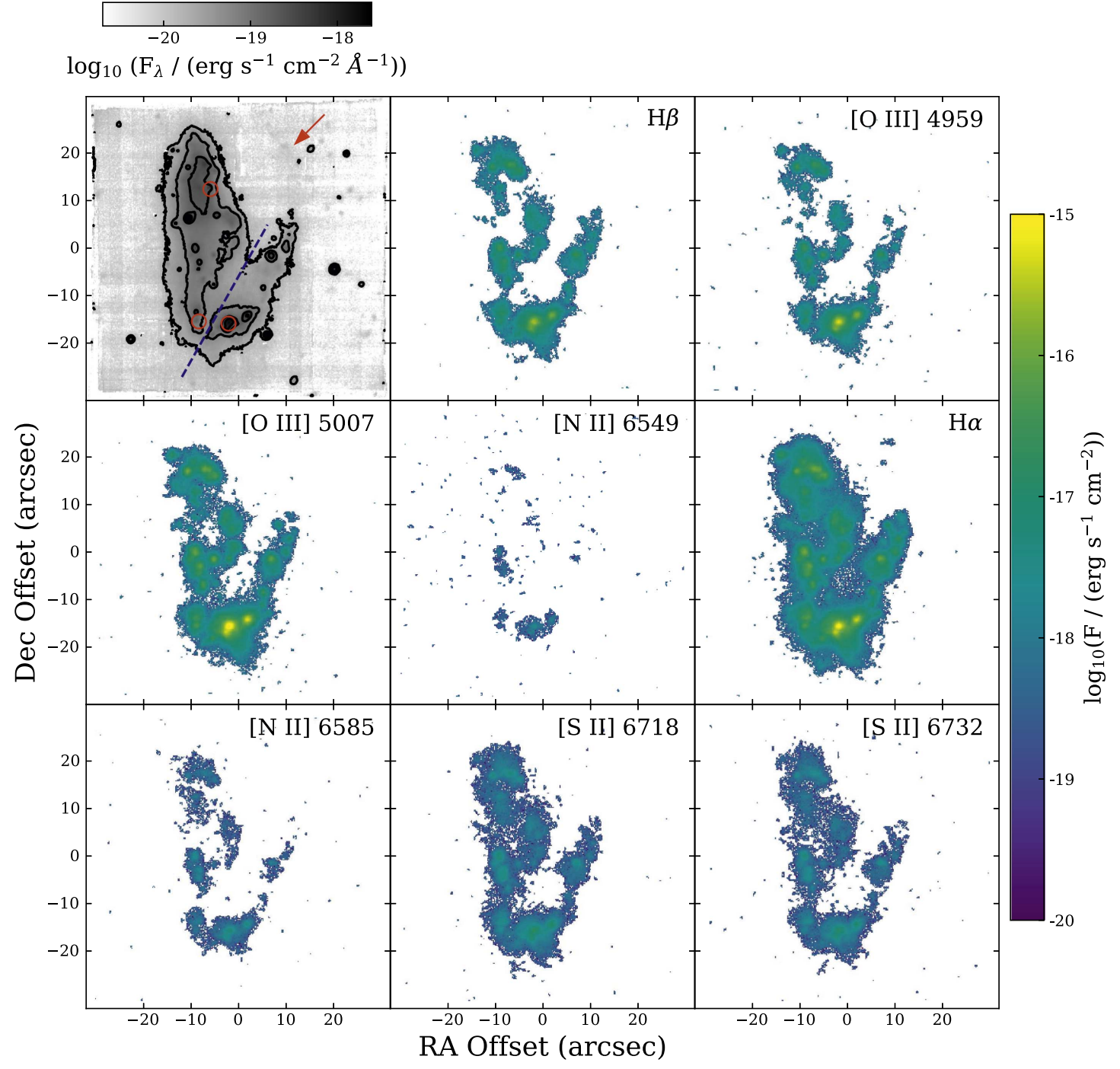

Figure 1. Images created from the MUSE data cube. Top row, from the left: $r$ broadband image, H $\beta$, and [O III] 4959. Middle row, from the left: [O III] 5007, [N II] 6549, and $\mathrm{H} \alpha$. Bottom row, from the left: [N II] 6585, [S II] 6718, and [S II] 6732. All images span the same field of view (1 arcmin, $11.3 \mathrm{kpc}$ on a side) centered on $16^{\mathrm{h}} 47^{\mathrm{m}} 10^{\mathrm{s}} .59+21^{\mathrm{d}} 05^{\mathrm{m}} 32^{\mathrm{s}} .15$. The red circles in the top left panel mark the approximate location and size of available SDSS fibers; the northern and southwestern fibers were used in the identification of the pair. The arrow in the top left panel marks the location of low-significance emission. This is suggestive of faint tidal debris extending from the southern galaxy and curving up toward the north, which is consistent with a tidal tail from the lower-mass galaxy. The H $\alpha$ image shows two clumps of emission at a similar location, which is further consistent with the possible identification of tidal material. The blue dashed line over the $r$-band image marks the approximate dividing line adopted to separate the two galaxies comprising the dm $1647+21$ system. The line images are all shown on the same logarithmic intensity scale and are not corrected for extinction.

Table 1

Line Detections

\begin{tabular}{lcc}
\hline \hline Line & $\begin{array}{c}\text { Integrated Flux } \\
\left(\times 10^{-15} \mathrm{erg} \mathrm{s}^{-1} \mathrm{~cm}^{-2}\right)\end{array}$ \\
\hline $\mathrm{H} \beta$ & 55.02 & 0.07 \\
{$[\mathrm{O} \mathrm{III]} 4959$} & 59.70 & 0.07 \\
{$[\mathrm{O} \mathrm{III]} \mathrm{5007}$} & 178.47 & 0.07 \\
{$[\mathrm{~N} \mathrm{II}] 5755$} & $<0.15$ & $\ldots$ \\
{$[\mathrm{N} \mathrm{II}] 6549$} & 0.94 & 0.04 \\
$\mathrm{H} \alpha$ & 189.22 & 0.05 \\
{$[\mathrm{~N} \mathrm{II}] 6585$} & 3.25 & 0.03 \\
{$[\mathrm{~S} \mathrm{II}] 6718$} & 11.30 & 0.03 \\
{$[\mathrm{~S} \mathrm{II}] 6732$} & 8.13 & 0.03 \\
\hline
\end{tabular}

Note. Line fluxes were measured from total intensity images (Figure 1) and errors were computed using the per-pixel variance values calculated during the pipeline reduction. The upper limit value quoted for [N II] 5755 is $3 \sigma$. the system. The two next most luminous clumps have extinctioncorrected fluxes of 10.6 and $9.9 \times 10^{-15} \mathrm{erg} \mathrm{s}^{-1} \mathrm{~cm}^{-2}$. Together these three clumps-all found in the lower-mass galaxy—account for $\sim 23 \%$ of the $\mathrm{H} \alpha$ luminosity. The remaining $~ 30$ clumps are distributed across both galaxies and each individually contribute less than $\sim 2 \%$ to the total flux; these clumps, and the remaining diffuse emission, effectively fill the same regions where we see stellar continuum emission. This clump analysis is based on visual identification of point-like emission in the $\mathrm{H} \alpha$ image; a more detailed analysis of the clumps in dm1647+21 and other systems will be presented by S. Liss et al. (2017, in preparation).

\subsubsection{On the Gas Depletion Time}

Single-dish H I observations of the system show a gas mass of $3.7 \times 10^{9} M_{\odot}$ (Stierwalt et al. 2015), however this measurement likely includes HI associated with a nearby 
system that is within the 8 arcminute GBT beam but not part of the interacting pair (UGC 10549, $z=0.009,50 \mathrm{kpc}$ projected separation). The integrated spectrum shows a somewhat complex profile, with the velocity of the peak emission at $\gtrsim 50 \mathrm{~km} \mathrm{~s}^{-1}$ lower velocity than the redshifts of the two components of $\mathrm{dm} 1647+21$. This peak corresponds to the redshift of UGC 10549, suggesting a substantial amount of the single-dish H I flux is associated with that galaxy.

In support of this interpretation of the single-dish profile, follow-up interferometric observations with the Very Large Array (VLA) reveal a clumpy $\mathrm{HI}$ distribution in dm1647+21 and emission associated with UGC 10549 (S. Stierwalt et al. 2017, in preparation). Unfortunately, the VLA observations (taken in the B-array) do not recover the total single-dish H I flux, so we cannot reliably separate the single-dish $\mathrm{H}$ I mass into contributions from $\mathrm{dm} 1647+21$ and UGC 10549. Considering the uncertain distribution of the $\mathrm{H}$ I mass, the single-dish measurement implies an upper limit to the gas depletion time of $2 \mathrm{Gyr}$ when considering only the SFR of dm1647+21. The true gas depletion time for $\mathrm{dm} 1647+21$ is likely shorter than this estimate.

\subsection{ISM Properties}

We use the line images created from the MUSE data to construct optical line ratio maps, based on classic optical diagnostic plots (e.g., Baldwin et al. 1981; Veilleux \& Osterbrock 1987; Kewley et al. 2006). Maps of $[\mathrm{O} \mathrm{III]} / \mathrm{H} \beta$, $[\mathrm{N} \mathrm{II}] / \mathrm{H} \alpha$, and $[\mathrm{S} \mathrm{II}] / \mathrm{H} \alpha$ are shown in Figure 2. The systemintegrated values for these ratios are: $\log _{10}([\mathrm{O} \mathrm{III}] / \mathrm{H} \beta)=0.51$, $\log _{10}([\mathrm{~N}$ II $] / \mathrm{H} \alpha)=-1.8$, and $\log _{10}([\mathrm{~S} \mathrm{II}] / \mathrm{H} \alpha)=-1.0$. In Figure 3, we show the line diagnostic diagrams with the distribution of values from Figure 2 and the source separation lines from Kewley et al. (2006). The line ratios in the ratio maps and diagnostic diagrams are all consistent with excitation from star formation, and we see no evidence for LINER-like or Seyfert emission.

Using the system-integrated [S II] line ratio of 1.40 , we estimate an upper limit to the electron density of $n_{\mathrm{e}}<10^{2} \mathrm{~cm}^{-3}$ (Osterbrock \& Ferland 2006). The spatial variation in this ratio is minimal in the regions with high signal to noise (Figure 2), suggesting consistently low $n_{\mathrm{e}}$ across the source. This low value may be due to a high-filling factor of low-density ionized gas and a low filling factor of young star clusters (which would have higher $n_{\mathrm{e}}$ densities).

We measure a system-integrated [N II] 6585/[N II] 6549 ratio of 3.5 , which is somewhat higher than the ratio fixed by quantum mechanics. This is likely due to the 6585 flux including regions where 6549 fell below the detection threshold, and artificially inflated the global ratio. The [N II] 5755 line is not detected, and we estimate a $3 \sigma$ upper limit of $0.15 \times 10^{-15} \mathrm{erg} \mathrm{s}^{-1} \mathrm{~cm}^{-2}$. Combining this with the sum of the two detected lines, the $[\mathrm{N}$ II] emission is consistent with a temperature of $T_{\text {nebula }}<20,000 \mathrm{~K}$.

\subsection{ISM Kinematics}

In Figure 4, we show the intensity-weighted line-of-sight velocity and dispersion for the $\mathrm{H} \alpha$ line. The images were constructed by Voronoi binning (e.g., Cappellari \& Copin 2003) the $\mathrm{H} \alpha$ line image (Figure 1) to a signal to noise of 50, including only individual pixels with $\geqslant 3 \sigma$. The resulting binning scheme was then applied to a continuum-subtracted data cube containing $\mathrm{H} \alpha$ and the intensity-weighted moment maps were computed from this binned cube. This velocity field is consistent with the pair separation of $27 \mathrm{~km} \mathrm{~s}^{-1}$, considering the locations of the SDSS fibers. The system shows a clear velocity gradient from north-to-south, spanning a total of $\sim 150 \mathrm{~km} \mathrm{~s}^{-1}$. The $\mathrm{H} \alpha$ velocity field for $\mathrm{dm} 1647+21$ shows irregular iso-velocity contours rather than the classic "spider" pattern of a smoothly rotating disk (e.g., Swaters et al. 2002, for dwarf galaxies). Due to the isolation of dm1647+21 from a nearby massive galaxy, external perturbation on a single object are unlikely to be the origin of this complex kinematic structure. The kinematics suggests the ionized gas is not part of a large, regularly rotating disk but instead are consistent with the system being a dynamical interaction of two dwarf galaxies.

The velocity dispersion is consistent with the instrumental resolution ( $\lesssim 100 \mathrm{~km} \mathrm{~s}^{-1}$ ), across the entire source, and we see no evidence for broadened lines that are indicative of shocks. This, as well as the line ratio maps (Figure 2), suggests that large-scale shocks from the ongoing merger are not contributing substantially to the energetics of the interstellar medium (ISM). ${ }^{7}$ If the line-of-sight velocities are representative (i.e., the bulk of the motion of these galaxies is not in the plane of the sky), they suggest a relatively slow encounter compared to major interactions between massive galaxies.

\section{4. dm1647+21 as a Dwarf-Dwarf Merger}

Based on the velocity field of $\mathrm{H} \alpha$ and optical morphology, $\mathrm{dm} 1647+21$ is consistent with being a pair of interacting dwarf galaxies. In the following two sub-sections, we briefly explore a "look-alike" dynamical model for the system and contrast the observed activity in dm1647+21 with the general characteristics of massive galaxy interactions.

\subsection{A “Look-alike” Numerical Model}

In the spirit of Toomre \& Toomre (1972), we created a "look-alike" dynamical model for this system, using the Identikit dynamical modeling tool (Barnes \& Hibbard 2009). While a bona fide dynamical model would need to be constrained by kinematics of tidal features or be verified by a simulation employing a self-gravitating disk, this look-alike model is instructive for qualitatively demonstrating the merger scenario for $\mathrm{dm} 1647+21$. We explored Identikit models corresponding to $2: 1$ mass ratio encounter on a parabolic orbit $(e=1)$. The galaxy models are the same as those used in Barnes \& Hibbard (2009) and Privon et al. (2013), with the scale lengths of the half-mass galaxy reduced by a factor $\sqrt{2}$ to maintain a constant mass surface density. While the observed stellar mass ratio of the two galaxies is 4.6:1, the baryonic mass ratio of the progenitors is uncertain owing to the likely dominant contribution of $\mathrm{H}$ I over the stellar mass. The lowermass galaxy is likely to have a higher gas fraction (e.g., Swaters et al. 2002; Bradford et al. 2015), implying a baryonic mass ratio that could be closer to $2: 1$.

We manually explored a range of initial galaxy orientations (the inclination of the disks relative to the orbital plane), time since first close pass, and viewing directions relative to the orbital plane. ${ }^{8} \mathrm{We}$ were able to reproduce the overall morphology and general sense of the velocity gradient using

\footnotetext{
7 Large-scale shocks are a significant contribution to the excitation of the ISM in mergers of massive galaxies (e.g., Rich et al. 2011, 2014).

8 For a detailed description of the Identikit modeling process, see Barnes \& Hibbard (2009) and Privon et al. (2013).
} 

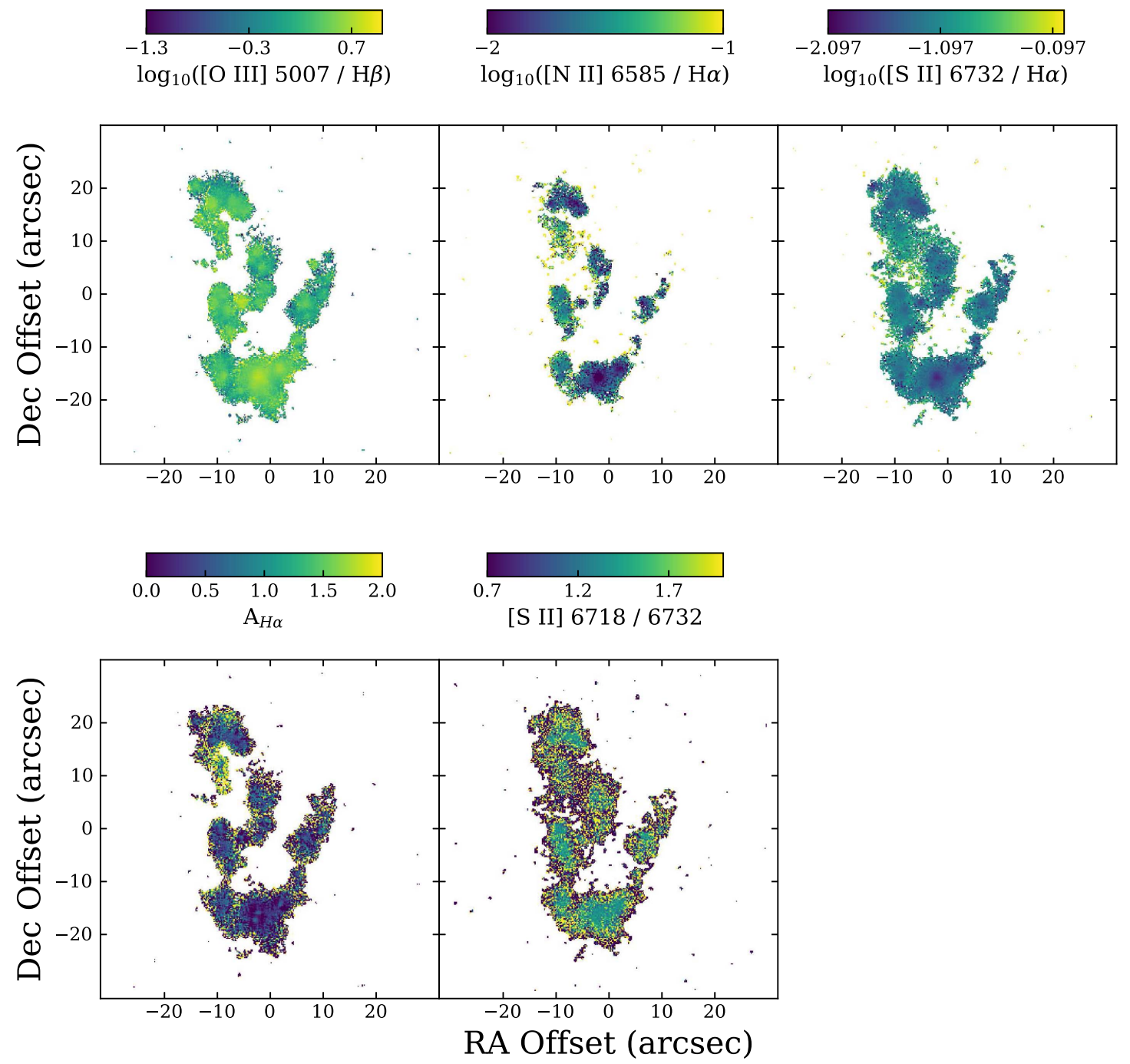

Figure 2. Optical line ratio images. First row, from left to right: $\left[\mathrm{O} \mathrm{III]} / \mathrm{H} \beta,[\mathrm{N} \mathrm{II}] / \mathrm{H} \alpha,[\mathrm{S} \mathrm{II}] / \mathrm{H} \alpha\right.$. Second row, from left to right: $A_{\mathrm{H} \alpha}$ and $[\mathrm{S}$ II] $6718 /[\mathrm{S}$ II] 6732 . The ratio maps were all masked to the fainter of the two lines (Figure 1). No extinction corrections have been applied.

a 2:1 mass ratio encounter where the more massive galaxy has a retrograde spin, the less massive galaxy has a prograde spin, and the system is being viewed after the first close pass of the two disks. In Figure 5, we show an overlay of the massless test particles in our look-alike model with the grayscale $r$-band image. The major features of the system can be qualitatively explained by this "look-alike" modeling, lending additional support to the identification of $\mathrm{dm} 1647+21$ as a pair of interacting dwarf galaxies. We additionally explored encounters with a total mass ratio of 4:1 (motivated by the observed stellar mass ratio) and were able to reproduce the observed morphology with a model in which both galaxies experience a prograde interaction. The morphology of the 4:1 look-alike is broadly consistent with that of the 2:1 mass ratio encounter, owing to the reduced tidal effect of the lower-mass galaxy on the massive companion. Distinguishing between these encounter scenarios will require detailed mapping of the total atomic gas distribution and subsequent detailed modeling of tidal features. Based on the small projected velocity range across the entire source, it seems likely these two dwarf galaxies are bound and will ultimately merge into a single dwarf.

In future TNT papers, we will explore detailed dynamical modeling of individual observed dwarf-dwarf interactions (including constraints from spatially resolved kinematics; S. Pearson et al. 2017, in preparation) as well as the properties of simulated paired and unpaired dwarfs in large-volume cosmological simulations (G. Besla et al. 2017, in preparation).

\subsection{Comparison with Massive Galaxy Mergers}

Stierwalt et al. (2015) showed that isolated dwarf pairs show star formation rates enhanced over isolated single dwarfs, with that enhancement increasing with decreasing pair separation. Massive galaxies show similar enhancements (Patton et al. 2013), however the SFR enhancements in massive mergers tend to occur in the nuclei (e.g., Armus et al. 1989; Mihos \& Hernquist 1994; Barnes \& Hernquist 1996), and star formation (SF) may even be suppressed on large scales due to the inflow of gas to the nucleus (Moreno et al. 2015).

In contrast, the star formation in $\mathrm{dm} 1647+21$ is both enhanced and distributed across the source, but not concentrated in one or two regions (i.e., there are many, distributed clumps and no single clump dominates the total SFR). The sSFR for this system is approximately a dex above that typical for dwarf galaxies in this mass range (sSFR $\sim 3 \times 10^{-10}$; Lee et al. 2011), which is consistent with the starburst identification of this system, based on the $\mathrm{H} \alpha \mathrm{EQW}$. Considering the lower-mass 


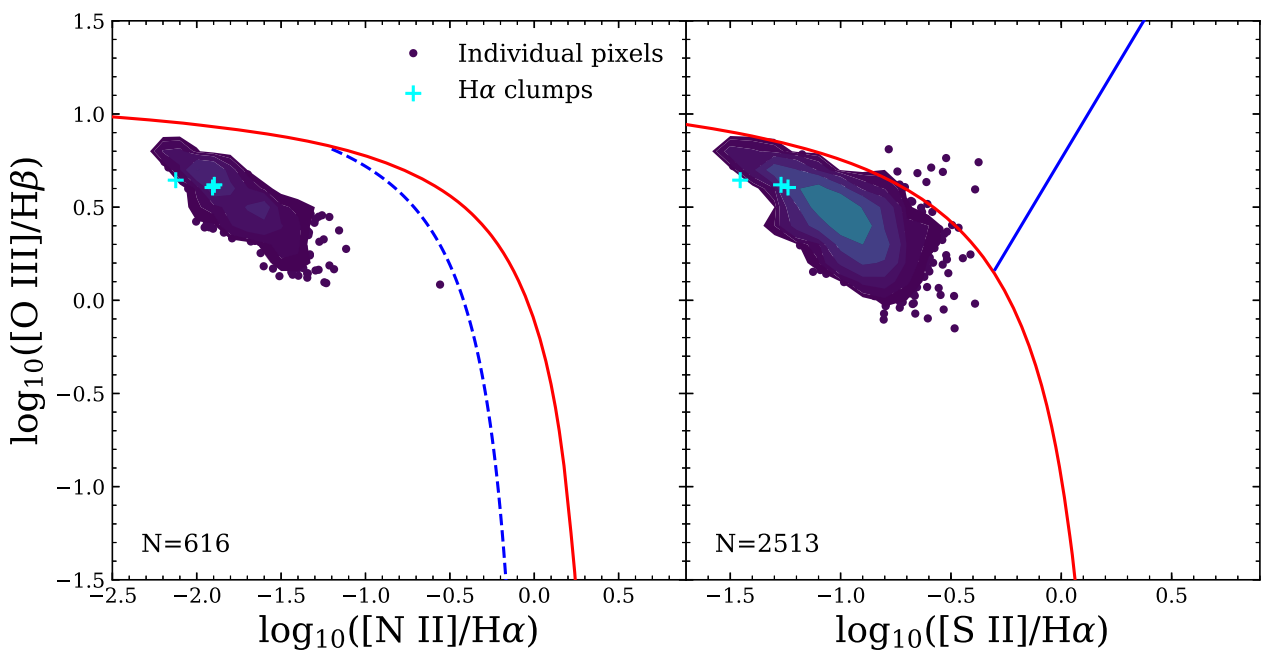

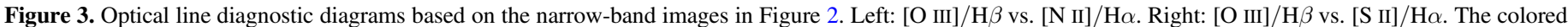

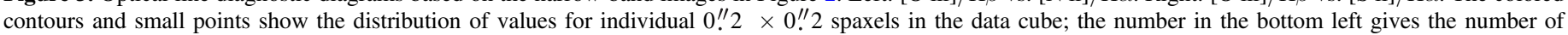

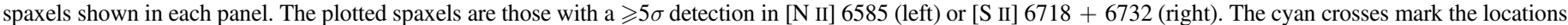

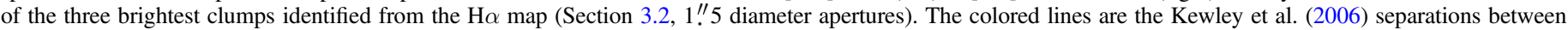
star-forming galaxies, Seyferts, and LINERs. The line ratios are consistent with excitation from ongoing star formation.

galaxy alone, its sSFR is a factor of $>50$ in excess of typical galaxies of that mass. So while the distributed, clumpy star formation is morphologically similar to other dwarf galaxies of similar mass, the significant enhancement points to the ongoing interaction as the likely driver of enhanced star formation in both galaxies. By comparison, the sSFR of dm1647+21 exceeds that of low-redshift infrared-selected luminous and ultraluminous infrared galaxies (Howell et al. 2010).

Why is the strongly enhanced star formation less concentrated in dm1647+21 compared to more massive galaxy mergers? A clue may lie in the typical H I properties of dwarfs. The baryonic mass of these dwarf pairs is dominated by the $\mathrm{H} \mathrm{I}$ (Stierwalt et al. 2015), and in a H I study of a nearby sample of dwarf pairs, Pearson et al. (2016) found a significant fraction (20\%-60\%) of the neutral atomic gas lies beyond the optical extent of these dwarf pairs. Extended H I distributions are also observed for non-interacting dwarf galaxies (Swaters et al. 2002). It thus seems generic for dwarf galaxies to have largescale H I envelopes.

Jog \& Solomon (1992) proposed a scenario for the triggering of star formation in galaxy merger overlap regions in which the collision of $\mathrm{H}$ I clouds increases the pressure in the cloud. The increased pressure within the $\mathrm{H}$ I envelope in turn triggers the collapse of pre-existing, formerly stable molecular clouds embedded within the $\mathrm{HI}$, resulting in a starburst. The elevated yet distributed starburst in $\mathrm{dm} 1647+21$ may be a consequence of the interaction of pre-existing $\mathrm{HI}$ envelopes of the two progenitor galaxies triggering the collapse of molecular clouds across the system. This is in contrast to massive galaxy mergers, where gas funneling to the nucleus is generally thought to precede the onset of the starburst (e.g., Barnes \& Hernquist 1991, 1996).

Thus, if dm1647+21 is a typical dwarf-dwarf merger, then the interactions enhance star formation but perhaps due to overpressuring of $\mathrm{HI}$ envelopes instead of funneling gas to the nuclei. A consequence of this is that the early encounter stages of dwarf interactions would not result in a significant build-up of mass in the nuclei and would instead result in a more distributed mass build-up. This, combined with the dissipational nature of the gas and the high gas fraction of the progenitor galaxies, suggests the remnant galaxy may retain or reform a dominant disk (e.g., Barnes 2004; Springel \& Hernquist 2005; Robertson et al. 2006; Robertson \& Bullock 2008) rather than becoming spheroid-dominated like mergers of massive galaxies. Consistent with this scenario, the Illustris-TNG hydrodynamic cosmological simulations do not find that the morphology of galaxies with $M_{*}<10^{11} M_{\odot}$ does not depend strongly on their merger history (Rodriguez-Gomez et al. 2017).

Simulations of low-mass galaxy mergers also show differences in the influence of mergers on low-mass galaxies compared to more massive galaxies. Kim et al. (2009) found distributed starbursts driven by shocks in high-resolution hydrodynamic simulations of low-mass, gas-rich galaxy mergers. Their inclusion of more detailed feedback treatment results in more well-defined starbursts in low-mass galaxies because the ISM becomes more strongly pressurized (Hopkins et al. 2013). This is in contrast to massive galaxies, where more realistic feedback treatment broadens and dilutes the starburst peak. Besla et al. (2012) argue that starbursts should be preferentially introduced in the lower-mass galaxy during dwarf-dwarf interactions. This is consistent with the brightest $\mathrm{H} \alpha$ knots and $60 \%$ of the total $\mathrm{H} \alpha$ flux residing to the lowermass component of $\mathrm{dm} 1647+21$, as well as its significantly higher sSFR. Using cosmological zoom simulations, Brooks \& Christensen (2016) find that simulated low-mass merger remnants have less mass in their centers than the progenitor galaxies; while it is not possible to discern if this type of mass rearranging is occurring in $\mathrm{dm} 1647+21$, our observations are in qualitative agreement with their simulations that the remnants of low-mass mergers may not be as centrally concentrated as remnants of massive galaxy mergers.

In idealized merger simulations representing massive highredshift galaxies, Fensch et al. (2017) found that higher gas fractions correspond to a reduced direct impact of the dynamical interaction on the star formation rate. For both non-interacting and interacting galaxies, simulated star formation rates were elevated for systems with higher gas fractions. However, the interacting system had fewer excursions above the main sequence of star formation and they were of reduced 


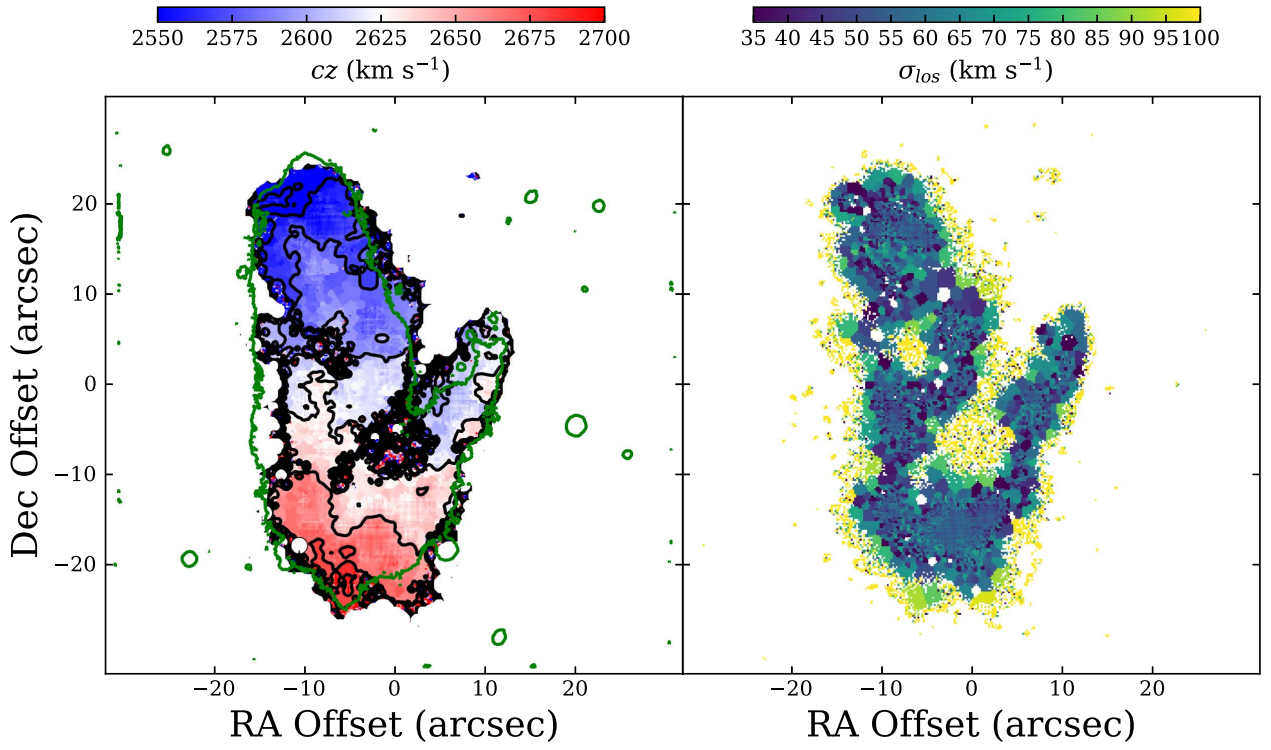

Figure 4. Left: intensity-weighted line-of sight velocity from the $\mathrm{H} \alpha$ emission shown as a function of position. The black contours are spaced at $25 \mathrm{~km} \mathrm{~s}{ }^{-1}$ intervals between 2550 and $2700 \mathrm{~km} \mathrm{~s}^{-1}$. The green contour is the lowest contour from the $r$ image in Figure 1. Right: intensity-weighted velocity dispersion of H $\alpha$, shown as a function of position. The velocity dispersion map is consistent with $\mathrm{H} \alpha$ being spectrally unresolved.

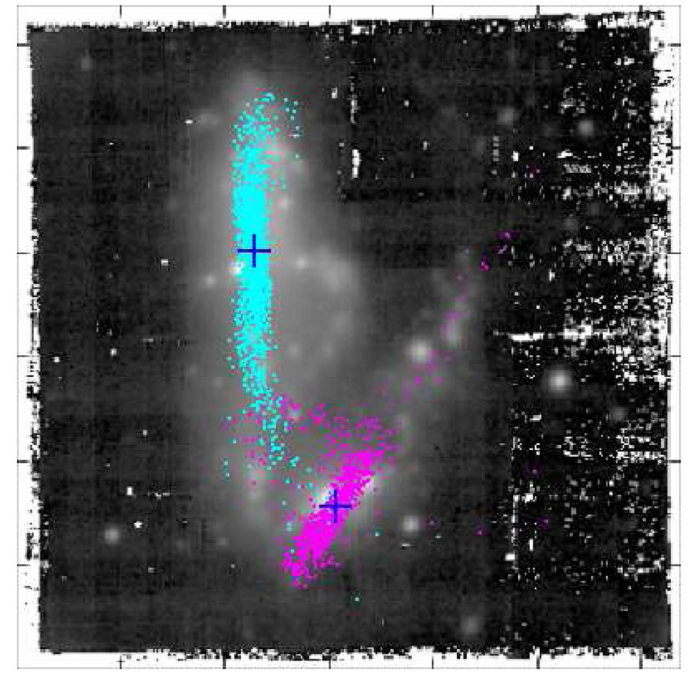

Figure 5. $r$-band image, overlaid with a look-alike dynamical model. The cyan and magenta points mark the positions of massless test particles from the disks of the two progenitor galaxies. The general morphology of the system can be explained by a 2:1 mass ratio with a retrograde (more massive galaxy; cyan) prograde (less massive galaxy; magenta) encounter being viewed shortly after the first close pass of the disks.

amplitudes compared to simulated interacting galaxies with lower gas fractions. They argue strong inflows driven by clumps in the (non-interacting) high gas fraction disk result in a reduced effectiveness of tidally induced torques to trigger the starburst because gas inflows are already prevalent in those massive galaxies.

In comparing to low-redshift dwarf galaxies, Fensch et al. (2017) note that, despite similar gas fractions to their simulations, non-interacting low-redshift dwarf galaxies have a reduced efficiency of inflows driven by disk instabilities and a more diffuse ISM at low-redshift. They argue this makes dwarf galaxy interactions at low-redshift more likely to result in SFR enhancements (compared to their high-redshift simulations) by driving inflows far in excess of those secularly driven in disks.
However, we argue here that the enhancement in dm1647+21 may not be the result of inflows, but rather large-scale ISM compression. Determining if torques are able to more efficiently drive gas to the center in later merger stages requires observations of other low-redshift, low-mass interacting systems.

\section{Conclusions}

We present VLT/MUSE IFU observations of the interacting dwarf pair dm1647+21. We detect the major optical diagnostic lines, which we use to demonstrate the excitation of the ISM can be fully explained by star formation. We also find the following.

1. By combining the $\mathrm{H} \alpha$ and $22 \mu \mathrm{m}$ detections, we measure a total star formation rate of $0.48 M_{\odot} \mathrm{yr}^{-1}$ (90\% of which is unobscured). This unobscured star formation is 2.7 times higher than inferred from extrapolations of SDSS spectroscopy.

2. The specific star formation for the system $(5.3 \times$ $10^{-9} \mathrm{yr}^{-1}$ ) is more than an order of magnitude higher than the sSFR for non-interacting galaxies in the same mass range and yet is distributed across the source. The sSFR for the lower-mass galaxy of the pair shows an even larger enhancement, which is consistent with the theoretical expectations that starbursts should preferentially be trigger in the lower-mass member of dwarf pairs.

3. The velocity structure, based on the $\mathrm{H} \alpha$ emission, is not consistent with an ordered, rotating disk.

4. Based on the kinematic and morphological evidence, and a "look-alike" numerical simulation, we conclude dm $1647+21$ is a pair of dynamically interacting dwarf galaxies. This dynamical interaction is the likely cause of the substantially enhanced star formation.

This system suggests important hydrodynamical differences from more massive galaxy interactions, including more widespread star formation and a lack of large-scale shocks. These SF properties may be explained by a scenario in which the 
pre-existing H I disks/envelopes of dwarf galaxies are compressed in the interaction, which leads to overpressure and collapse of previously stable dense clumps of gas throughout the galaxy. This suggests that mergers affect the mass build-up of dwarf galaxies in a way that is different from massive galaxies. In the future, we will expand this study to other dwarf pairs in the TNT survey to assess how representative this object is of dwarf-dwarf interactions as class.

The authors thank the anonymous referee for their comments, which have improved the quality of the paper. The authors thank F.E. Bauer for access to computing facilities which were used to process the MUSE data.

G.C.P. was supported by a FONDECYT Postdoctoral Fellowship (No. 3150361). D.R.P. was supported by a Discovery Grant from NSERC of Canada. N.K. is supported by the NSF CAREER award 1455260. S.E.L. acknowledges support from the NSF GRFP (Grant No. DDGE-1315231), the Virginia Space Grant Consortium, and the Clare Boothe Luce Foundation.

The authors thank the Sexten Center for Astrophysics (http://www.sexten-cfa.eu) where part of this work was performed. This research has made use of the NASA/IPAC Extragalactic Database (NED) and NASA's Astrophysics Data System.

Based on observations collected at the European Organisation for Astronomical Research in the Southern Hemisphere under ESO programme 097.B-0504(A). This research has used data from the Sloan Digital Sky Survey. Funding for the Sloan Digital Sky Survey IV has been provided by the Alfred P. Sloan Foundation, the U.S. Department of Energy Office of Science, and the Participating Institutions. SDSS acknowledges support and resources from the Center for High-performance Computing at the University of Utah. The SDSS web site is www.sdss.org. This publication makes use of data products from the Wide-field Infrared Survey Explorer, which is a joint project of the University of California, Los Angeles, and the Jet Propulsion Laboratory/California Institute of Technology, funded by the National Aeronautics and Space Administration.

Facility: VLT:Yepun (MUSE).

Software: ESO Reflex (Freudling et al. 2013), ipython (Pérez \& Granger 2007), numpy (Van Der Walt et al. 2011), matplotlib (Hunter 2007), Astropy (Astropy Collaboration et al. 2013), Identikit (Barnes \& Hibbard 2009, 2010), Zeno (Barnes 2011).

\section{ORCID iDs}

G. C. Privon (ib https://orcid.org/0000-0003-3474-1125

D. R. Patton (1) https://orcid.org/0000-0002-1871-4154

S. Pearson (i) https://orcid.org/0000-0003-0256-5446

K. E. Johnson (1) https://orcid.org/0000-0001-8348-2671

\section{References}

Armus, L., Heckman, T. M., \& Miley, G. K. 1989, ApJ, 347, 727

Astropy Collaboration, Robitaille, T. P., Tollerud, E. J., et al. 2013, A\&A, 558, A 33
Bacon, R., Accardo, M., Adjali, L., et al. 2010, Proc. SPIE, 7735, 773508

Baldwin, J. A., Phillips, M. M., \& Terlevich, R. 1981, PASP, 93, 5

Barnes, J. E. 1988, ApJ, 331, 699

Barnes, J. E. 2004, MNRAS, 350, 798

Barnes, J. E. 2011, ZENO: N-body and SPH Simulation Codes, Astrophysics Source Code Library, ascl:1102.027

Barnes, J. E., \& Hernquist, L. 1996, ApJ, 471, 115

Barnes, J. E., \& Hernquist, L. E. 1991, ApJL, 370, L65

Barnes, J. E., \& Hibbard, J. E. 2009, AJ, 137, 3071

Barnes, J. E., \& Hibbard, J. E. 2010, Identikit 1: A Modeling Tool for Interacting Disk Galaxies, Astrophysics Source Code Library, ascl: 1011.001

Besla, G., Kallivayalil, N., Hernquist, L., et al. 2012, MNRAS, 421, 2109

Bradford, J. D., Geha, M. C., \& Blanton, M. R. 2015, ApJ, 809, 146

Brinchmann, J., Charlot, S., White, S. D. M., et al. 2004, MNRAS, 351, 1151

Brooks, A., \& Christensen, C. 2016, Bulge Formation via Mergers in Cosmological Simulations, Astrophysics and Space Science Library, Vol. 418 (Dordrecht: Springer), 317

Cappellari, M., \& Copin, Y. 2003, MNRAS, 342, 345

Chang, Y.-Y., van der Wel, A., da Cunha, E., \& Rix, H.-W. 2015, ApJS, 219, 8

Duc, P.-A., Cuillandre, J.-C., Karabal, E., et al. 2015, MNRAS, 446, 120

Fensch, J., Renaud, F., Bournaud, F., et al. 2017, MNRAS, 465, 1934

Freudling, W., Romaniello, M., Bramich, D. M., et al. 2013, A\&A, 559, A96

Gordon, K. D., Clayton, G. C., Misselt, K. A., Landolt, A. U., \& Wolff, M. J. 2003, ApJ, 594, 279

Hinshaw, G., Larson, D., Komatsu, E., et al. 2013, ApJS, 208, 19

Hopkins, P. F., Cox, T. J., Hernquist, L., et al. 2013, MNRAS, 430, 1901

Hopkins, P. F., Hernquist, L., Cox, T. J., \& Kereš, D. 2008, ApJS, 175, 356

Howell, J. H., Armus, L., Mazzarella, J. M., et al. 2010, ApJ, 715, 572

Hunter, J. D. 2007, CSE, 9, 90

Jog, C. J., \& Solomon, P. M. 1992, ApJ, 387, 152

Kennicutt, R. C. 1998, ARA\&A, 36, 189

Kewley, L. J., Groves, B., Kauffmann, G., \& Heckman, T. 2006, MNRAS, 372, 961

Kim, J.-H., Wise, J. H., \& Abel, T. 2009, ApJL, 694, L123

Lee, J. C., Gil de Paz, A., Kennicutt, R. C., Jr., et al. 2011, ApJS, 192, 6

Lee, J. C., Gil de Paz, A., Tremonti, C., et al. 2009a, ApJ, 706, 599

Lee, J. C., Kennicutt, R. C., Jr., Funes, S. J. J. G., Sakai, S., \& Akiyama, S. 2009b, ApJ, 692, 1305

Maraston, C., Strömbäck, G., Thomas, D., Wake, D. A., \& Nichol, R. C. 2009, MNRAS, 394, L107

Mihos, J. C., \& Hernquist, L. 1994, ApJL, 431, L9

Moreno, J., Torrey, P., Ellison, S. L., et al. 2015, MNRAS, 448, 1107

Osterbrock, D. E., \& Ferland, G. J. 2006, Astrophysics of Gaseous Nebulae and Active Galactic Nuclei (2nd ed.; Sausalito, CA: University Science Books)

Patton, D. R., Torrey, P., Ellison, S. L., Mendel, J. T., \& Scudder, J. M. 2013, MNRAS, 433, L59

Pearson, S., Besla, G., Putman, M. E., et al. 2016, MNRAS, 459, 1827

Pérez, F., \& Granger, B. E. 2007, MCSE, 9, 21

Privon, G. C., Barnes, J. E., Evans, A. S., et al. 2013, ApJ, 771, 120

Rich, J. A., Kewley, L. J., \& Dopita, M. A. 2011, ApJ, 734, 87

Rich, J. A., Kewley, L. J., \& Dopita, M. A. 2014, ApJL, 781, L12

Robertson, B., Bullock, J. S., Cox, T. J., et al. 2006, ApJ, 645, 986

Robertson, B. E., \& Bullock, J. S. 2008, ApJL, 685, L27

Rodriguez-Gomez, V., Sales, L. V., Genel, S., et al. 2017, MNRAS, 467, 3083

Sanders, D. B., Soifer, B. T., Elias, J. H., et al. 1988, ApJ, 325, 74

Springel, V., \& Hernquist, L. 2005, ApJL, 622, L9

Stierwalt, S., Armus, L., Surace, J. A., et al. 2013, ApJ, 206, 1

Stierwalt, S., Besla, G., Patton, D., et al. 2015, ApJ, 805, 2

Stierwalt, S., Liss, S. E., Johnson, K. E., et al. 2017, NatAs, 1, 0025

Swaters, R. A., van Albada, T. S., van der Hulst, J. M., \& Sancisi, R. 2002, A\&A, 390, 829

Toomre, A., \& Toomre, J. 1972, ApJ, 178, 623

Tremonti, C. A., Heckman, T. M., Kauffmann, G., et al. 2004, ApJ, 613, 898 Van Der Walt, S., Colbert, S. C., \& Varoquaux, G. 2011, arXiv:1102.1523

Veilleux, S., \& Osterbrock, D. E. 1987, ApJS, 63, 295

Wright, E. L., Eisenhardt, P. R. M., Mainzer, A. K., et al. 2010, AJ, 140, 1868 